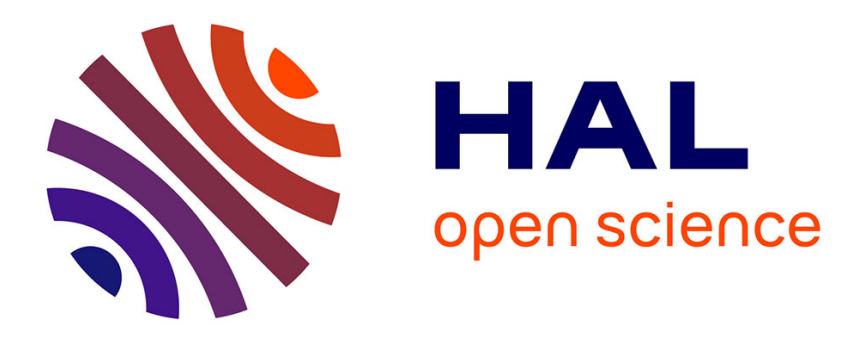

\title{
Politiques éducatives et évasion fiscale dans les pays en développement
}

Florence Arestoff, Jean-François Jacques

\section{To cite this version:}

Florence Arestoff, Jean-François Jacques. Politiques éducatives et évasion fiscale dans les pays en développement. Revue d'Economie Politique, 2016, 126 (6), pp.1057-1075. 10.3917/redp.266.1057 . hal-02877015

\section{HAL Id: hal-02877015 \\ https://hal.science/hal-02877015}

Submitted on 1 Jul 2020

HAL is a multi-disciplinary open access archive for the deposit and dissemination of scientific research documents, whether they are published or not. The documents may come from teaching and research institutions in France or abroad, or from public or private research centers.
L'archive ouverte pluridisciplinaire HAL, est destinée au dépôt et à la diffusion de documents scientifiques de niveau recherche, publiés ou non, émanant des établissements d'enseignement et de recherche français ou étrangers, des laboratoires publics ou privés. 


\section{Politiques éducatives et évasion fiscale dans les pays en développement}

Octobre 2011, version révisée juillet 2014, cette révision octobre 2015

Florence ARESTOFF ${ }^{1}$ et Jean-François JACQUES ${ }^{2}$

Résumé : L'objectif de cet article est de mettre en évidence les conséquences que peut avoir l'évasion fiscale sur la durée de la scolarisation et les dépenses allouées à l'éducation. Nous ne faisons pas d'hypothèse particulière sur l'origine de l'évasion fiscale. Nous supposons simplement une société composée de deux catégories d'agents hétérogènes, les riches et les pauvres. Parmi les riches, certains déclarent l'intégralité de leur revenu alors que d'autres déclarent percevoir le revenu des pauvres. Faute de moyens de contrôle, l'Etat n'est pas apte à différencier les riches fraudeurs et les pauvres. De plus, nous introduisons des complémentarités dans la fonction d'accumulation de capital humain en termes de financement de l'éducation. Notre modèle est décliné en deux cas correspondant chacun à des politiques éducatives différentes c'est-à-dire des modes différents de financement de l'éducation. Dans un régime public, l'Etat collecte des impôts pour financer l'éducation et, dans un régime mixte (privé-public), l'Etat finance l'éducation par l'impôt tandis que les parents les plus riches peuvent compléter ce financement de manière privée. Nous montrons que la taxation dépend positivement de l'ampleur de l'évasion fiscale mais que cette sous-optimalité peut être compensée, dans le régime mixte, par une durée de scolarisation obligatoire plus importante.

Mots-clés : Financement de l'éducation, Développement, Evasion fiscale. JEL : I25, I28, H26.

\footnotetext{
${ }^{1}$ PSL, Université Paris-Dauphine, LEDa, DIAL UMR225, F-75016 Paris, France; IRD, LEDa, DIAL UMR225, F-75010, Paris, France. Adresse mail : florence.arestoff@dauphine.fr.

${ }^{2}$ Université Paris-Est, ERUDITE (EA 437), UPEM 77454 Marne la Vallée, France; PSL, Université Paris-Dauphine, LEDa-CGEMP. Adresse mail : JeanFrancois.Jacques@u-pem.fr

Nous remercions José Scheinkman pour ses encouragements à travailler sur ce thème, Antoine d'Autume, les deux rapporteurs ainsi que l'éditeur pour leurs remarques constructives sur les versions précédentes de ce papier. Ce travail a par ailleurs bénéficié de nombreux commentaires lors de présentations à la Journée d'Etudes du GDR Economie du développement et de la transition du CERDI (2008, Clermont-Ferrand), à la conférence Ecomod (2008, Berlin), au colloque du GDRI DREEM (2009, Istanbul) et au séminaire du LEDa-Dauphine. Nous en remercions tous les participants. Toute erreur qui pourrait subsister dans le papier serait biensûr de notre entière responsabilité.
} 


\title{
Education policies and tax evasion in developing countries
}

\begin{abstract}
The goal of this article is to highlight the consequences that tax evasion could impact on schooling duration and expenditures in education. We don't make any specific assumptions as regards the origin of the tax evasion. We simply consider a society made up of two categories of heterogeneous agents, the rich and the poor. Among the rich people, some declare their total income whereas the others declare the same income as the poor. While the government is unable to control this factor, it can't distinguish the wealthy tax evaders from the poor ones either. Moreover, in the human capital accumulation function, we have introduced complementarities between personal tax contributions. Our model is declined in two cases, each corresponding to different education policies characterized by different ways of education funding. In the public education regime, the government collects income tax. Tax revenues are used to finance education. In the mixed education regime (public and private), the government finances education by collecting income tax whereas the richest parents can complete this education privately. We show that taxation depends positively on how large the tax evasion is but we also show that, in the mixed education regime, this sub optimality can be corrected by a longer compulsory length of schooling.

Keywords: Education financing, Development, Tax evasion.

JEL : I25, I28, H26.
\end{abstract}




\section{Introduction}

L'éducation est un élément essentiel du développement économique et social constituant un moyen d'accroître directement le bien-être de la population et de favoriser la croissance économique. De fait, l'éducation a toujours occupé une place de choix dans les domaines d'intervention de l'Etat, y compris dans les régions les plus pauvres. Ainsi, en 2010, les pays d'Afrique Subsaharienne allouent $4.4 \%$ de leur PIB aux dépenses publiques d'éducation, soit plus que les pays d'Asie du Sud (2.8\%) par exemple (World Bank, 2015). Pourtant, c'est en Afrique Subsaharienne que les taux de scolarisation de la population sont les plus faibles. Parmi les différentes raisons justifiant une telle situation, la forte contrainte financière qui pèse sur les Etats africains est souvent avancée. Or cette forte contrainte peut être renforcée par un phénomène d'évasion fiscale. Dans les pays en développement, l'évasion fiscale s'explique, selon Ahmad et Stern (1989), par cinq raisons essentiellement. La première, la plus immédiate, est que le taux d'imposition est jugé trop élevé par la population. La deuxième provient du poids des activités illégales dans l'économie. Ces activités génèrent des revenus, qui, par définition ne sont pas soumis à l'imposition d'où un taux de taxation moyen plus faible que le taux légal. La troisième raison tient à la multiplication de petites unités de production artisanales qui s'avèrent difficiles à contrôler. La quatrième raison est qu'une forte part de la production, notamment agricole, est une production domestique, largement échangée sous forme de troc donc là encore non soumise à l'impôt. Enfin la dernière raison soulignée par Tanzi (1983) revient à considérer la prohibition et la corruption bureaucratique, voire, plus généralement, le manque de développement des institutions, comme un facteur clef de l'évasion fiscale. Ces raisons pourraient aussi être invoquées pour des pays développés mais prennent un relief particulier dans les pays en développement. Il convient alors de s'interroger sur les conséquences de l'évasion fiscale sur les décisions que peuvent prendre l'Etat ou les familles en termes d'éducation.

Ici, nous ne faisons pas d'hypothèse particulière sur l'origine de l'évasion fiscale. Nous supposons simplement une société composée de deux catégories d'agents hétérogènes, les riches et les pauvres. Parmi les riches, certains déclarent l'intégralité de leur revenu alors que d'autres déclarent percevoir le revenu des pauvres. L'Etat sait que la population est constituée de riches et de pauvres mais n'ayant pas (ou trop peu) de moyens de contrôle, il n'est 
pas apte à identifier les riches et les pauvres.

Nous proposons un modèle à générations imbriquées avec deux catégories d'agents. Ce type de modèle avec accumulation de capital a été introduit par Diamond (1965). Glomm (1997) l'a repris pour comparer l'efficacité des systèmes publics et privés d'éducation en termes de croissance. Nous introduisons des complémentarités dans la fonction d'accumulation de capital humain en termes de financement de l'éducation. Ces complémentarités jouent un rôle essentiel dans nos résultats.

Notre modèle est décliné en plusieurs cas correspondant chacun à des politiques éducatives différentes c'est-à-dire des modes différents de financement de l'éducation. Ainsi, dans un régime public, l'Etat intervient dans le domaine de l'éducation et, tel un planificateur social, se positionne à l'état stationnaire pour déterminer à la fois la durée des études de la jeune génération et les dépenses scolaires, sources de qualité scolaire. Parallèlement, dans un régime mixte (privé-public), l'Etat finance le système scolaire par l'impôt tandis que les parents les plus riches complètent l'éducation de manière individuelle et privée, en payant par exemple des cours complémentaires à leurs enfants.

En raison de l'évasion fiscale qui met l'Etat dans une situation de moindres recettes face à l'assiette fiscale dont il dispose, nous ne comparons que des optima de second rang. Notre objectif est alors de mettre en évidence les conséquences que peut avoir l'évasion fiscale, mesurée ici par la taille de la population qui ne déclare pas ses revenus effectifs, sur la durée de la scolarisation et les dépenses allouées à l'éducation. Nous montrons en particulier qu'une durée de scolarisation obligatoire plus importante peut, sous certaines hypothèses, combler le manque de rentrées fiscales.

La section 2 du papier présente les hypothèses du modèle. La section 3 détermine les solutions obtenues pour des politiques éducatives privées ou publiques. Dans la section 4, nous spécifions le cas hybride d'une éducation privée complétant l'éducation publique financée par l'impôt. La conclusion du papier fait l'objet de la section 5 .

\section{Le modèle}

Soit une économie à générations imbriquées dans laquelle les agents, qu'il soient riches ou pauvres, vivent deux périodes, jeune et adulte. Au cours de 
la seconde période de vie, chaque individu donne naissance à un autre individu ce qui assure une population constante dans le temps dont la taille est normalisée à un. Chaque génération correspond à un continuum d'agents. Chacun est doté d'une unité de temps. En première période de vie, un jeune alloue son temps entre éducation et exercice d'un emploi non qualifié. En deuxième période de vie, les adultes offrent leur travail de manière inélastique. Ils exercent des activités qualifiées en utilisant le capital humain accumulé quand ils étaient jeunes. Dans le cadre d'un pays en développement où les systèmes de retraite font défaut, les individus exercent une activité professionnelle à tout âge. Les modèles tels que celui de Barham et alii (1995), qui supposent une dernière période de vie consacrée à la retraite nous semblent donc peu réalistes.

Plus précisément, l'économie est composée d'individus indicés par $j$ qui appartiennent à deux classes d'agents, les agents pauvres $(i=1)$ et les agents riches $(i=2)$.

A chaque date $t$, il existe trois biens dans l'économie : le travail, le capital humain et un bien de consommation produit en quantités $y_{t}$. La technologie de production est à rendements d'échelle constants et nécessite le travail des enfants et des parents. Les parents consacrent la totalité de leur temps disponible (soit l'unité) à travailler et les enfants y consacrent une partie seulement, l'autre étant dédiée à la formation. A une date $t$, la technologie de production dépend alors du capital humain des parents qu'ils ont accumulé pendant leur jeunesse en $(t-1)$ et qui devient disponible en $t, h_{t}$, et de la fraction de temps que les enfants consacrent au travail, $n_{t}$. Les enfants ne disposent pas de capital humain parce que celui-ci n'est pas immédiatement valorisé; il ne sera utilisable qu'à la période suivante. Ces deux types de travail étant imparfaitement substituables, la technologie de production peut être décrite par une fonction Cobb-Douglas dans laquelle $A$ est un paramètre de productivité. Nous supposons que l'Etat, confronté à un phénomène d'évasion fiscale, a connaissance de la totalité des revenus de la population mais ne connaît pas l'identité des contribuables. Plus précisément, l'Etat sait qu'il existe deux niveaux de productivité $A$ dans l'économie, constantes dans le temps : $A_{1}$ (productivité des pauvres) et $A_{2}$ (productivité des riches) avec $A_{2}>A_{1}$. Mais il ne sait pas ex ante qui sont les pauvres et qui sont les riches.

Pour chaque individu $j$ du groupe $i$, la fonction de production familiale 
s'écrit :

$$
y_{j i t}=A_{i} h_{j i t}^{\beta} n_{j i t}^{1-\beta}
$$

$0<\beta<1$

Le choix d'une fonction de production de forme Cobb-Douglas repose sur le fait que le concept de division familiale du travail ne signifie pas que les activités de chacun soient totalement séparées. En réalité, la plupart d'entre elles s'imbriquent les unes avec les autres. Dans le cas des pays en développement, le secteur informel est prédominant. Les enquêtes 1-2-3 menées entre 2001 et 2003 auprès d'échantillons de ménages représentatifs de sept capitales de pays de l'UEMOA (Union Economique et Monétaire des pays Ouest Africains) montrent ainsi que le pourcentage de chefs de ménage qui tirent leur revenu principal du secteur informel varie de $47 \%$ à Niamey (Niger) à $61 \%$ à Lomé $(\text { Togo })^{3}$. Or, les études sur le dualisme du marché du travail urbain ont permis de caractériser le secteur informel par de petites entreprises domestiques. Comme le montre Pourcet (1995, p.202), les membres de la famille pratiquent diverses activités complémentaires dans le but d'approvisionner régulièrement et suffisamment le budget du ménage. Par conséquent, les enfants travaillent en compagnie de l'un ou l'autre de leurs parents et non indépendamment d'eux.

Dans le cas où les jeunes exercent une activité professionnelle en même temps qu'ils suivent des études, comme nous le supposons dans le modèle, il semble aussi pertinent de considérer que parents et enfants travaillent ensemble. Ainsi, dans son analyse du secteur informel au Mexique, Roubaud (1994) montre que la durée des études pour des jeunes appartenant à des familles pauvres, engagées dans l'informel, est souvent conditionnée à leur participation à l'unité de production familiale.

Nous préférons donc une fonction de production familiale de type CobbDouglas plutôt que celle retenue par Glomm (1997) qui suppose que $h_{t}$ et $n_{t}$ sont parfaitement substituables pour $y_{t}$.

Du point de vue des préférences, celles des parents sont définies sur leur consommation de biens $c_{j i t}$ et le capital humain futur de leurs enfants $h_{j i, t+1}$. Ceci est le reflet d'un altruisme imparfait de la part des parents que l'on retrouve dans Behrman et al. (1982) mais aussi Galor et Zeira (1993), SaintPaul et Verdier (1993) et Epple et Romano (1998). Cette spécification a

\footnotetext{
${ }^{3}$ Voir notamment Böhme et Thiele, 2011.
} 
l'avantage de la simplicité. Elle montre que les parents se préoccupent du bien-être de leurs enfants au travers de certains critères spécifiques (tels que l'éducation) mais sans savoir a priori comment les enfants valoriseront ces critères. Ces préférences parentales sont aussi représentées par une fonction Cobb-Douglas, de même forme pour les individus $j$ des deux classes $i$ d'agents, riches et pauvres :

$$
U\left(c_{j i t}, h_{j i, t+1}\right)=c_{j i t}^{\alpha} h_{j i, t+1}^{1-\alpha}
$$

$0<\alpha<1$,

$(1-\alpha)$, le degré d'altruisme inter-générationnel ${ }^{4}$.

Enfin, en ce qui concerne l'accumulation de capital humain, pour chaque individu $j$ appartenant à la classe sociale $i$, le capital humain accumulé à la date $t$ pour la période suivante $h_{j i, t+1}$ dépend du temps que les enfants allouent à l'éducation $\left(1-n_{j i t}\right)$ ainsi que du capital humain accumulé par la génération précédente $h_{j i t}$ (dont on suppose qu'il ne se déprécie pas). Ces facteurs d'accumulation du capital humain sont standards dans la littérature sur la croissance avec capital humain. Ainsi, Bénabou (1996) considère que le capital humain des parents représente les capacités génétiques dont les enfants héritent. Conformément à la théorie du capital humain, le capital humain $h_{j i t}$ que les parents eux-mêmes transmettent à leurs enfants sous forme de legs intergénérationnel est supposé différent selon le milieu social.

A l'instar de Glomm (1997) et Glomm et Ravikumar (1992), nous supposons que l'accumulation de capital humain repose aussi sur le financement de l'éducation $e_{j i t}$, ce financement étant à l'origine de la qualité scolaire dont bénéficient les enfants. Nous considérons que cette qualité scolaire intègre deux aspects : l'un quantitatif, pris en compte par le montant individuel de financement, et l'autre, qualitatif, pris en compte par la complémentarité des contributions individuelles. Celles-ci sont formalisées par le produit et non la somme de toutes les contributions. Ces complémentarités jouent un rôle essentiel dans les résultats. Le planificateur cherchera à les promouvoir via le taux de taxe notamment pour conduire à une accumulation optimale du capital humain. Le financement de l'éducation est donc collectif, représenté par une moyenne géométrique de la contribution financière des parents pauvres et de celle des parents riches. Le paiement des parents riches ne peut

\footnotetext{
${ }^{4}$ Pour une présentation plus complète, voir Gérard-Varet, L.A., Kolm S.C. and J. Mercier Ythier (ed) (2000)
} 
se substituer totalement au paiement des parents pauvres, il en est partiellement complémentaire. La contribution de chaque classe sociale est à la fois spécifique et indispensable aux autres.

Dans une population adulte de taille $N$, les parents pauvres sont en quantité $N_{1}$ tandis que les riches sont en quantité $N_{2}$. Leurs différences de productivité se traduisent alors par des revenus différents $y_{j i t}$ donc par des capacités à financer les dépenses scolaires $e_{j i t}$ elles-mêmes différentes. Enfin, le capital humain est accumulé selon une technologie de type Cobb-Douglas généralisé.

Par conséquent, pour un individu $j$ de la classe $i$, la fonction d'accumulation de capital humain s'écrit :

$$
h_{j i, t+1}=\theta\left(1-n_{j i t}\right) \cdot h_{j i t}^{\delta}\left(\prod_{j=1 . . N} e_{j t}\right)^{\gamma / N}
$$

On ne considèrera que des situations symétriques dans lesquelles tous les pauvres se comportent de manière identique ainsi que tous les riches.

Pour les individus pauvres $i=1$, la fonction d'accumulation de capital humain devient donc, à l'équilibre :

$$
h_{1 t+1}=\theta\left(1-n_{1 t}\right) \cdot h_{1 t}^{\delta}\left(e_{1 t}^{N_{1}} \cdot e_{2 t}^{N_{2}}\right)^{\gamma / N}
$$

Symétriquement, pour les individus riches de la catégorie $i=2$, cette fonction d'accumulation devient :

$$
h_{2 t+1}=\theta\left(1-n_{2 t}\right) \cdot h_{2 t}^{\delta}\left(e_{1 t}^{N_{1}} \cdot e_{2 t}^{N_{2}}\right)^{\gamma / N}
$$

$\theta>0$

$0 \leq \delta, \gamma \leq 1$

Le capital humain transmis par les parents à leurs enfants $\left(h_{1}\right.$ ou $\left.h_{2}\right)$ est une externalité associée à une élasticité $\delta$, de même que les dépenses scolaires dont l'effet externe a pour élasticité le paramètre $\gamma$. Le paramètre $\theta$ mesure l'efficacité de la production du capital humain. Cette efficacité est supposée constante dans le temps et entre les individus car fonction de facteurs exogènes tels que les facilités individuelles, le voisinage, les caractéristiques du système scolaire, etc. 


\section{Régime d'éducation public ou privé}

Dans le régime privé, la société s'organise et l'économie fonctionne sans qu'il y ait besoin de gouvernement. Les parents inscrivent donc forcément leurs enfants dans des écoles privées financées par leurs soins. Dans le régime public au contraire, l'offre d'éducation provient uniquement du système scolaire public. L'Etat se charge de collecter des impôts afin de financer l'éducation. Nous supposons que la technologie de production de l'éducation est équivalente d'un régime à l'autre d'où, pour un même budget de fonctionnement, une efficacité identique des écoles publiques et privées.

\subsection{Le régime privé ou l'impossibilité du laissez-faire}

Dans le régime privé, les agents choisissent la durée de scolarité $\left(1-n_{t}\right)$ de leurs enfants et le montant des ressources allouées à l'éducation de leurs enfants $e_{t}$ de manière à maximiser le bien-être individuel. La qualité de l'éducation reçue par chaque enfant est alors uniquement fonction de la richesse de ses propres parents.

Pour chaque individu $j$ de la classe $i^{5}$, le revenu familial est partagé entre la consommation et les dépenses scolaires de la manière suivante :

$$
e_{j i t}=y_{j i t}-c_{j i t}
$$

Notons que dans la théorie standard du capital humain notamment exposée par Ben-Porath (1967), l'individu lui-même décide d'investir ou non dans sa formation en fonction du taux de rendement anticipé. Or, un certain nombre de raisons rendent inadéquate la possibilité d'une prise de décision individuelle. D'une part, la durée d'études moyenne ne va souvent pas audelà du primaire dans de nombreux pays d'Afrique. Comme le note Glomm (1997), "(il semble logique) de supposer que toutes les décisions scolaires seront prises par les parents pour les enfants et que les enfants devront accepter ces décisions". D'autre part, s'éduquer suppose de faire face à deux

\footnotetext{
${ }^{5}$ Il convient de préciser qu'en régime privé, au sein d'une classe d'agents (pauvres ou riches), les individus $j$ sont supposés différents ex-ante. En revanche, à l'équilibre donc ex-post, ils deviennent identiques au sein de leur classe car nous considérons des équilibres symétriques.
} 
types de coûts : le coût direct et le coût d'opportunité de l'éducation. Sous l'hypothèse d'un marché des capitaux fonctionnant parfaitement, les jeunes qui ne possèdent pas personnellement ces fonds peuvent les emprunter. Or, dans un pays en développement, cette possibilité n'est pas réaliste : soit les banques refuseront directement de prêter à un jeune en l'absence de garanties suffisantes, soient elles refuseront indirectement en lui proposant des taux d'intérêt usuraires (Becker, 1975, 1981). Pour s'éduquer, le recours au financement parental s'avère donc nécessaire.

Raisonnons ici pour une classe sociale $i$ donnée, les pauvres par exemple pour lesquels $i=1$.

En $t$, les parents $j$ qui sont pauvres résolvent le programme suivant :

$$
\begin{aligned}
\underset{n_{j 1 t}, e_{j 1 t}}{\operatorname{Max} U\left(c_{j 1 t}, h_{j 1, t+1}\right)} & =c_{j 1 t}^{\alpha} h_{j 1, t+1}^{1-\alpha} \\
s / c y_{j 1 t} & =A_{1} h_{j 1 t}^{\beta} n_{j 1 t}^{1-\beta} \\
c_{j 1 t} & =y_{j 1 t}-e_{j 1 t} \\
h_{j 1, t+1} & =\theta\left(1-n_{j 1 t}\right) \cdot h_{j 1 t}^{\delta} \cdot\left(e_{j 1 t}^{N_{1}} \cdot e_{j 2 t}^{N_{2}}\right)^{\gamma / N}
\end{aligned}
$$

avec $n_{j 1 t} \in[0,1], h_{j 1 t}>0, c_{j 1 t}>0, e_{j 1 t}, e_{j 2 t}>0$

Cela revient à maximiser la fonction

$$
U=\left[A_{1} h_{j 1 t}^{\beta} n_{j 1 t}^{1-\beta}-e_{j 1 t}\right]^{\alpha}\left[\theta\left(1-n_{j 1 t}\right) \cdot h_{j 1 t}^{\delta} \cdot\left(e_{j 1 t}^{N_{1}} \cdot e_{j 2 t}^{N_{2}}\right)^{\gamma / N}\right]^{1-\alpha}
$$

Les parents pauvres considèrent $h_{j 1 t}$ donné puisque le capital humain des parents résulte uniquement de la scolarisation qui a eu lieu pendant leur jeunesse en $(t-1)$.

Par ailleurs, les agents étant atomistiques, la contribution de chacun au financement de l'éducation est négligeable car le terme $\left(e_{j 1 t}^{N_{1}} \cdot e_{j 2 t}^{N_{2}}\right)$ est considéré comme exogène. Par conséquent, la maximisation de l'utilité relativement à $e_{j 1 t}$ conduit inexorablement à un financement nul de l'éducation :

$$
\left(\frac{e_{j 1 t}}{y_{j 1 t}}\right)=0
$$

Sachant qu'en situation de laissez-faire, cette part du revenu consacrée à l'éducation est la même pour les ménages riches et pour les ménages pauvres, 
$\left(\frac{e_{j 1 t}}{y_{j 1 t}}\right)=\left(\frac{e_{j 2 t}}{y_{j 2 t}}\right)=0$, on retrouve le problème du financement privé d'un bien public. Chacun paie en fonction de son utilité marginale ce qui crée un problème d'efficacité économique.

En l'absence de financement de l'éducation, l'accumulation de capital humain devient lui-même nul. C'est pourquoi le laissez-faire constitue ici un cas impossible. De fait, aucun pays au monde ne dispose d'un régime d'éducation qui soit exclusivement privé. L'Etat contribue toujours au financement de l'éducation, que ce soit totalement ou partiellement.

\subsection{Le régime public}

Dans le régime public, l'éducation est exclusivement dispensée au sein d'écoles publiques qui offrent une qualité scolaire homogène en termes de fonctionnement et de construction des écoles. De même que Saint-Paul et Verdier (1993), Fernandez et Rogerson (1995) ou Epple et Romano (1996a, 1996b et 1998) par exemple, nous considérons que le financement de l'éducation des jeunes provient d'une taxation sur le revenu des ménages. Dans le cas d'un pays en développement où le recours à la taxation du revenu est très limité (Bourguignon, 2000) car reposant essentiellement sur les salaires des fonctionnaires et des employés des entreprises modernes, cette hypothèse peut sembler discutable. Dans ces pays, les droits de douane représentent encore une grande partie des revenus fiscaux même si l'imposition des biens de consommation (TVA par exemple) se développe largement ${ }^{6}$. Ici, cependant, taxer la consommation revient à taxer le revenu puisque l'investissement en éducation $(e)$ est intégralement réalisé par l'Etat.

Si l'Etat finance l'éducation par la taxation des revenus, la taille de l'assiette fiscale devient prépondérante. Or, dans de nombreux pays en développement, un impôt sur le revenu qui repose sur l'auto-déclaration ne peut pas être contrôlé, faute de ressources administratives (Slemrod and Yitzhaki, 2002). En présence d'évasion fiscale, l'assiette fiscale est alors mal évaluée par l'Etat.

En effet, l'Etat connaît le paramètre de productivité A dans la fonction de production familiale. Il sait que $\mathrm{A}$ appartient à l'ensemble $\left\{A_{1}, A_{2}\right\}, A_{1}$ étant le paramètre de productivité des pauvres et $A_{2}$, celui des riches. Mais

\footnotetext{
${ }^{6} \mathrm{~A}$ titre illustratif, elle est passée de $25 \%$ à plus de $40 \%$ des recettes fiscales du Mali entre 1995 et 2000 (Gunther et al., 2007).
} 
il n'a pas les moyens d'identifier ex ante les parents riches et les parents pauvres. Il ne connaît que le nombre d'individus qui se déclarent riche et le nombre d'individus qui se déclarent pauvre. Dit autrement, l'Etat connait l'ampleur de la fraude mais il ne connaît pas l'identité des fraudeurs. On supposera alors qu'il maximise le bien-être des agents les plus pauvres.

Plus précisément, nous supposons désormais que la population adulte $\mathrm{N}$ se décompose en trois catégories d'individus :

- a individus pauvres et se déclarant comme tels

- b individus riches mais se déclarant pauvre auprès des services de l'Etat

- c individus riches et se déclarant comme tels.

S'il existe des familles à forte productivité qui déclarent l'ensemble de leurs revenus, c'est peut-être parce que leur activité est publique et connue de tous. L'hypothèse d'un contrôle social est implicite ici ${ }^{7}$.

En lien avec le modèle précédent, cette décomposition revient à écrire : $N_{1}=a$ et $N_{2}=b+c$.

Comme Basdevant (1999), nous considérons que l'Etat, en tant que planificateur social, décide de la durée de formation (ou du temps de travail) de la jeune génération, ainsi que du taux d'imposition permettant le financement de l'éducation. Contrairement aux parents, l'Etat a conscience qu'un enfant est un futur parent. Il investit donc davantage dans l'éducation que ne le feraient les parents qui n'ont comme horizon de calcul que la période suivante.

Conformément au critère de justice sociale de Rawls, l'Etat se préoccupe du bien-être des plus pauvres $(i=1)$ et cherche donc $n$ et $\tau$ qui maximisent le bien-être individuel à l'état stationnaire. Il raisonne alors à $h_{2}$ et $e_{2}$ donnés pour résoudre le programme suivant :

$$
\begin{aligned}
\underset{n_{1}, \tau}{\operatorname{Max} U\left(c_{1}, h_{1}\right)} & =c_{1}^{\alpha} h_{1}^{1-\alpha} \\
s / c y_{1} & =A_{1} h_{1}^{\beta} n_{1}^{1-\beta} \\
c_{1} & =(1-\tau) y_{1} \\
h_{1} & =\theta\left(1-n_{1}\right) \cdot h_{1}^{\delta}\left[\left(\tau y_{1}\right)^{a+b} \cdot\left(\bar{\tau} y_{2}\right)^{c}\right]^{\gamma / N}
\end{aligned}
$$

\footnotetext{
${ }^{7}$ En régime public, contrairement au régime privé, l'Etat considère d'emblée, ex-ante, que tous les individus d'une même catégorie (pauvre, riche honnête ou riche malhonnête) sont identiques.
} 
Faute d'informations sur leur identité, l'Etat assimile les riches qui se déclarent pauvres (en nombre $b$ ) à des pauvres (en nombre $a$ ) et leur applique un taux d'imposition commun $\tau$. L'existence de cette catégorie d'agents riches qui se déclarent pauvres conduit naturellement à de moindres recettes fiscales donc à un moindre financement de l'éducation. L'Etat applique en revanche un taux d'imposition plus élevé, $\bar{\tau}$, aux riches qui déclarent l'intégralité de leur revenu. En supposant qu'il existe un taux d'imposition maximal $\tau_{\max }$, en raison de contraintes non explicitées, l'Etat va choisir ce taux maximal pour les riches qui se déclarent comme tels $\left(\bar{\tau}=\tau_{\max }\right)$. Notons que si ce taux maximal $\tau_{\max }$ n'existait pas, l'Etat fixerait un taux d'imposition $\bar{\tau}=1$ car il ne se préoccupe que du bien-être du pauvre et le bien-être du pauvre dépend de la contribution totale du riche.

De plus, le programme de maximisation étant le même pour les riches et les pauvres, l'Etat est conduit à déterminer un niveau d'études optimal $(1-n)$ tel que $n_{1}=n_{2}=n$. De ce fait, les agents riches ou pauvres ne choisissent pas la durée d'études $(1-n)$ qui est déterminée plus loin. Elle est obligatoire et identique pour tous les agents. L'offre de travail $n$ est donc identique pour tous quel que soit $\bar{\tau}$ (et quel que soit $\tau$ ).

Sous ces conditions, le programme de maximisation de l'utilité peut se réécrire :

$\underset{n, \tau}{M a x} U\left(c_{1}, h_{1}\right)=(1-\tau)^{\alpha} A_{1}^{\alpha} n^{\alpha(1-\beta)}\left[\theta(1-n)\left(\tau A_{1}\right)^{\frac{(a+b) \gamma}{N}} \cdot\left(\bar{\tau} A_{2}\right)^{\frac{c \gamma}{N}} n^{(1-\beta) \gamma}\right]^{\frac{\alpha \beta+(1-\alpha)}{1-\delta-\gamma \beta}}$

Les conditions de premier ordre conduisent à l'expression de $\tau$ telle que :

$$
\tau=\frac{\gamma(\alpha \beta+1-\alpha)(a+b)}{\alpha N(1-\delta-\gamma \beta)+\gamma(a+b)(\alpha \beta+1-\alpha)}
$$

Le taux d'imposition $\tau$ est une fonction croissante de $(a+b)$. De fait, en raison des effets externes de l'éducation, l'Etat impose d'autant plus les pauvres (ou assimilés) qu'ils sont nombreux. Ce résultat repose sur l'élasticité du rendement éducatif de l'impôt, c'est-à-dire $\frac{(a+b) \gamma}{N}$. Le mécanisme des complémentarités entre les contributions individuelles joue ici pleinement son effet : plus le nombre d'agents contribuant à la qualité scolaire est grand, plus leurs contributions se renforcent mutuellement. Dans l'intérêt de l'agent pauvre, l'Etat va alors s'appuyer sur ces complémentarités pour augmenter le capital 
humain via la contribution fiscale des pauvres. Ainsi, comme l'efficacité est une moyenne géométrique, plus $(a+b)$ est élevé plus le nombre des individus que l'Etat considère comme pauvres augmente et plus le rendement de l'impôt est lui-même élevé. Ceci conduit l'Etat à prélever davantage de taxes sur les pauvres (qu'ils le soient vraiment ou pas).

L'évasion fiscale $(b>0)$ élève donc le taux d'imposition $\tau$ ce qui joue en défaveur des individus pauvres (en quantités $a$ ) et crée une sous-optimalité, les riches fraudeurs étant insuffisamment taxés.

On déduit ensuite l'expression de $n$ telle que :

$$
\frac{\partial U}{\partial n}=0 \Leftrightarrow \frac{\alpha(1-\beta)}{n}=\frac{\alpha \beta+1-\alpha}{1-\delta-\gamma \beta}\left[\frac{1}{1-n}-\frac{(1-\beta) \gamma}{n}\right]
$$

Le terme de gauche en $n$ traduit l'utilité marginale tirée de la consommation et le terme de droite en $(1-n)$ l'utilité marginale tirée du legs de capital humain à la génération suivante. Rappelons en effet que $n$ correspond au temps que les jeunes consacrent à travailler et $(1-n)$ au temps qu'ils consacrent à étudier. Selon le terme de gauche, une augmentation de $n$ permet d'accroître le revenu familial donc permet aux parents de consommer davantage, d'où une utilité marginale décroissante de la consommation. Selon le terme de droite, plus $(1-n)$ augmente et plus les enfants font d'études donc plus ils sont à même d'accumuler du capital humain, source de bien-être pour leurs parents. Cet effet est renforcé par l'externalité du bien public $\gamma$ : plus $\gamma$ est élevé et plus l'utilité du capital humain s'accroît

L'Etat est donc confronté à un arbitrage entre la consommation des parents et le capital humain légué.

De cette expression, on déduit :

$$
n=\frac{(1-\beta)[\alpha(1-\delta)+\gamma(1-\alpha)]}{1-(1-\beta)[\gamma(1-\alpha)-\alpha \delta]}
$$

Le fait que $0<n<1$ confirme que les parents souhaitent que leurs enfants étudient $(n<1)$. Toutefois, en raison de la substituabilité imparfaite des facteurs dans la fonction de production familiale mais aussi en raison $\mathrm{du}$ fait que toutes les fonctions soient log additivement séparables, tout le temps disponible aux enfants ne peut pas être consacré aux études $(n>0)^{8}$.

\footnotetext{
${ }^{8}$ La baisse de revenu qu'induit un accroissement de la durée des études des enfants traduit le coût d'opportunité de l'éducation, dont le poids dans l'investissement en capital humain a été mis en évidence par Schultz (1963).
} 
Il apparaît ici que le temps que les jeunes consacrent à travailler est constant et indépendant de $h$ le stock de capital humain parental. Cela signifie que la distribution de richesse dans la population n'a pas d'effet sur l'allocation du temps entre éducation et travail. Bien que ce résultat semble discutable, il est habituel dans la littérature [Lucas (1988) et Azariadis et Drazen (1992), par exemple]. De même, $n$ est indépendant des différentes catégories de population $(a, b, c)$ mais aussi du paramètre de productivité $A$ qui distingue les riches et les pauvres. Ce résultat est lié à la forme Cobb-Douglas des fonctions, forme la plus couramment retenue dans cette littérature mais aussi au fait que l'élasticité de $n$ est constante si on suppose constante la taille de la population $N$.

En revanche, l'Etat prend en considération l'externalité du capital humain $\delta$ dans le calcul de $n$. Toute augmentation de $\delta$ se traduit par une baisse de $n$ donc un accroissement de la durée d'études $(1-n)$. En contrepartie, moins les enfants sont éduqués (donc plus $n$ est élevé), plus le revenu familial est élevé (effet de $\beta$ ), et plus le revenu familial est élevé, plus les parents peuvent consommer ce qui vient accroître leur bien-être (effet de $\alpha$ ).

\section{Régime public et éducation complémentaire privée}

Regardons maintenant ce qui se passerait dans un régime public laissant la possibilité aux parents de fournir à leurs enfants une éducation personnalisée complémentaire à celle de l'école publique. Seuls les parents riches peuvent fournir cet effort financier complémentaire. Mais il sera de montant différent selon que ces parents riches sont honnêtes ou pas.

\subsection{L'éducation complémentaire}

Il convient de rappeler que la population adulte à la date $t$ comprend désormais trois catégories d'agents $i$ : les agents pauvres $(i=1)$, les agents riches mais se déclarant pauvres auprès des services de l'Etat $(i=2)$ et les agents riches qui ne le cachent pas $(i=3)$.

Soit $\bar{F}$ le financement complémentaire des parents qui sont à la fois riches (leur productivité $A$ est forte et égale à $A_{3}$ tel que $A_{3}=A_{2}$ ) et honnêtes. Leur taux d'imposition est alors $\bar{\tau}$. 
Soit $F$ le financement complémentaire des parents qui sont riches mais dont les revenus ne sont pas intégralement déclarés ce qui leur permet de se voir appliquer le même taux d'imposition que la catégorie des plus pauvres, c'est-à-dire $\tau$.

Les parents de la catégorie $(i=3)$ vont déterminer le montant de leur financement complémentaire $\bar{F}$ en raisonnant dans un équilibre "à la Stackelberg", l'Etat étant leader. L'individu $j$ appartenant à la catégorie d'agents $i=3$ détermine donc $\overline{F_{j}}$ avec $h_{j 3 t}, \tau, \bar{\tau}$ et $n$ donnés. A l'équilibre, tous les individus de la catégorie $i=3$ sont identiques mais ex-ante, dans le programme de maximisation, les individus $j$ sont supposés différents. De plus, ils n'ont pas de comportement intertemporel au delà de la période suivante.

Le programme de maximisation s'écrit alors :

$$
\begin{aligned}
\operatorname{Max}_{\overline{F_{j}}} U\left(c_{j 3 t}, h_{j 3, t+1}\right) & =c_{j 3 t}^{\alpha} h_{j 3, t+1}^{1-\alpha} \\
s / c y_{j 3 t} & =A_{3} h_{j 3 t}^{\beta} n^{1-\beta} \\
c_{j 3 t} & =(1-\bar{\tau}) y_{j 3 t}-e_{\overline{F j}} \\
h_{j 3, t+1} & =\theta(1-n) \cdot h_{j 3 t}^{\delta} \cdot\left[\left(\tau y_{1 t}\right)^{a} \cdot\left(\tau y_{2 t}\right)^{b}\left(\bar{\tau} y_{3 t}\right)^{c}\right]^{\gamma / N} \bar{F}_{j}^{\gamma}
\end{aligned}
$$

On pose $\bar{F}_{j}=1$ si $e_{\overline{F j}}=0$ et $\overline{F_{j}}=e_{\overline{F j}}$ si $e_{\overline{F j}}$ est différent de zéro ce qui traduit un prolongement naturel du modèle précédent.

Cette formulation de la fonction $h_{j 3, t+1}$ permet de considérer que les parents riches peuvent payer des cours particuliers à leurs enfants ou leur offrir des manuels scolaires supplémentaires afin que les enfants puissent accumuler davantage de capital humain par des moyens annexes et individuels. En revanche, ce financement complémentaire ne peut pas modifier la qualité scolaire offerte dans les écoles publiques, homogène pour tous les élèves et financée par l'impôt. De ce fait, pour les parents riches et honnêtes, le terme $\left[\left(\tau y_{1 t}\right)^{a} \cdot\left(\tau y_{2 t}\right)^{b}\left(\bar{\tau} y_{3 t}\right)^{c}\right]^{\gamma / N}$ qui représente la qualité scolaire, est considéré comme donné. C'est l'éducation publique partagée par tous et financée par tous, contrairement au financement privé $\overline{F_{j}}$ qui ne profite qu'à l'individu lui-même et ne constitue pas une externalité ${ }^{9}$. Par conséquent, même si à

\footnotetext{
${ }^{9}$ Indirectement, la collectivité toute entière profite néanmoins de ce financement complémentaire car des enfants mieux éduqués percevront de plus hauts revenus à l'âge adulte donc contribueront davantage, à l'état stationnaire, au financement de l'éducation par l'impôt.
} 
l'état stationnaire, le revenu $y_{2}$ des parents riches et malhonnêtes dépend de leur financement complémentaire $(F)$, nous supposons que les parents riches et honnêtes $(i=3)$ n'internalisent pas cet effet car ils considèrent qu'ils n'ont aucune prise sur les termes entre crochets, pris comme des données.

Par ailleurs, nous faisons ici l'hypothèse simplificatrice que nos catégories d'agents demeurent dans leur catégorie d'une génération à l'autre. Cette hypothèse simplificatrice nous permet d'avoir des résultats analytiques. Comment y remédier? On pourrait par exemple supposer que des agents changent de catégorie d'une manière aléatoire d'une génération à l'autre. Mais il existerait toujours trois catégories d'agents pour lesquels les comportements en termes de financement complémentaire de l'éducation resteraient semblables à ceux que nous identifions ici. On peut donc penser, et nous l'espérons, que les résultats obtenus ne seraient pas fondamentalement modifiés si nous levions cette hypothèse.

La résolution du programme de maximisation conduit à la solution suivante :

$$
\bar{F}_{j}=\frac{\gamma(1-\alpha)(1-\bar{\tau})}{\alpha+\gamma(1-\alpha)} \cdot y_{j 3 t}
$$

Sachant que $y_{j 3 t}=A_{3} h_{j 3 t}^{\beta} n^{1-\beta}$, plus les parents sont éduqués ( $h_{j 3 t}$ élevé)

et plus ils dépensent pour l'éducation complémentaire de leurs enfants. On retrouve la conclusion de Becker and Tomes (1976) selon laquelle une augmentation du revenu des parents devrait engendrer une relative augmentation des dépenses destinées aux enfants. La part de ce financement complémentaire dans le revenu $\left(\bar{F}_{j} / y_{j 3 t}\right)$ est par ailleurs d'autant plus élevée que l'élasticité des dépenses scolaires dans la fonction d'accumulation de capital humain est importante ( $\gamma$ élevé). De même, plus l'altruisme parental est important $[(1-\alpha)$ élevé $]$, plus $\left(\overline{F_{j}} / y_{j 3 t}\right)$ est grand. En revanche, $\delta$ l'élasticité du capital humain initial dans la fonction d'accumulation n'a pas d'effet. Les parents n'internalisent pas l'externalité positive engendrée par l'héritage de capital humain d'une génération à l'autre.

Pour déterminer $F_{j}$, l'individu $j$ appartenant à la catégorie d'agents $i=2$ résoud un programme de même forme conduisant à un financement complémentaire qui vaut : 


$$
F_{j}=\frac{\gamma(1-\alpha)(1-\tau)}{\alpha+\gamma(1-\alpha)} \cdot y_{j 2 t}
$$

Dans un second temps, puisqu'hormis ce financement complémentaire privé, le régime d'éducation reste public, l'Etat va choisir $n, \tau$ et $\bar{\tau}$ connaissant $\bar{F}$.

Dans son calcul d'optimisation, l'Etat ne tient pas compte des agents riches qui ne déclarent pas tout leur revenu $(i=2)$. Il se doute que les riches malhonnêtes vont eux aussi compléter l'éducation de leurs enfants de manière personnelle, pour un montant $F$. Mais, comme l'Etat n'est pas apte à les identifier, il les assimile aux agents pauvres.

D'après le critère rawlsien, l'Etat va donc chercher à maximiser l'utilité du pauvre c'est-à-dire

$$
\underset{n, \tau, \bar{\tau}}{\operatorname{Max}} U\left(c_{1}, h_{1}\right)=c_{1}^{\alpha} h_{1}^{1-\alpha}
$$

Comme le financement de l'éducation est une moyenne géométrique reposant sur la contribution de l'ensemble de la collectivité, l'Etat sait que le capital humain des agents pauvres est une fonction de celui des riches.

Or, pour chaque catégorie d'agents, la fonction d'accumulation du capital humain s'écrit comme suit, à l'état stationnaire :

$$
\begin{aligned}
& h_{1}=\theta(1-n) \cdot h_{1}^{\delta} \cdot\left[\left(\tau y_{1}\right)^{a} \cdot\left(\tau y_{2}\right)^{b}\left(\bar{\tau} y_{3}\right)^{c}\right]^{\gamma / N} \\
& h_{2}=\theta(1-n) \cdot h_{2}^{\delta} \cdot\left[\left(\tau y_{1}\right)^{a} \cdot\left(\tau y_{2}\right)^{b}\left(\bar{\tau} y_{3}\right)^{c}\right]^{\gamma / N} F^{\gamma} \\
& h_{3}=\theta(1-n) \cdot h_{3}^{\delta} \cdot\left[\left(\tau y_{1}\right)^{a} \cdot\left(\tau y_{2}\right)^{b}\left(\bar{\tau} y_{3}\right)^{c}\right]^{\gamma / N} \bar{F}^{\gamma}
\end{aligned}
$$

Mais l'Etat ne distinguant pas les pauvres des riches malhonnêtes, il considère seulement :

$$
\begin{aligned}
& h_{1}=\theta(1-n) \cdot h_{1}^{\delta} \cdot\left[\left(\tau y_{1}\right)^{a+b} \cdot\left(\bar{\tau} y_{3}\right)^{c}\right]^{\gamma / N} \\
& h_{3}=\theta(1-n) \cdot h_{3}^{\delta} \cdot\left[\left(\tau y_{1}\right)^{a+b} \cdot\left(\bar{\tau} y_{3}\right)^{c}\right]^{\gamma / N} \bar{F}^{\gamma}
\end{aligned}
$$

Il en déduit une relation entre $h_{1}$ et $h_{3}$ :

$$
h_{3}=h_{1} \cdot \bar{F}^{\frac{\gamma}{1-\delta}}
$$


Sachant que $\bar{F}=\frac{\gamma(1-\alpha)(1-\bar{\tau})}{\alpha+\gamma(1-\alpha)} \cdot y_{3}$ et que la fonction de production familiale s'écrit $y_{i}=A_{i} h_{i}^{\beta} n_{i}^{1-\beta}$ quel que soit $i=1,2,3$, on en déduit que la dynamique du capital humain s'écrit :

$h_{1 t+1}=\theta(1-n) . h_{1 t}^{\delta}\left\{\left(\tau A_{1} n^{1-\beta} h_{1 t}^{\beta}\right)^{a+b}\left[\bar{\tau} A_{2} n^{1-\beta} h_{1 t}^{\frac{\beta(1-\delta)}{1-\delta-\gamma \beta}}\left(\frac{\gamma(1-\alpha)(1-\bar{\tau})}{\alpha+\gamma(1-\alpha)} A_{2} \cdot n^{1-\beta}\right)^{\frac{\beta \gamma}{1-\delta-\gamma \beta}}\right]^{c}\right\}^{\frac{\gamma}{N}}$

A l'état stationnaire, le capital humain de l'agent pauvre vaut alors :

$h_{1}=\theta(1-n) \cdot\left\{\tau^{a+b} \cdot \bar{\tau}^{c} n^{1-\beta\left[a+b+c \frac{(1-\delta)}{1-\delta-\gamma \beta}\right]} \cdot(1-\bar{\tau})^{\frac{c \gamma \beta}{1-\delta-\gamma \beta}} \cdot A_{1}^{a+b} \cdot A_{2}^{c \frac{(1-\delta)}{1-\delta-\gamma \beta}} \cdot\left(\frac{\gamma(1-\alpha)}{\alpha+\gamma(1-\alpha)}\right)^{\frac{c \gamma \beta}{1-\delta-\gamma \beta}}\right\}^{X}$

avec $X=\frac{\gamma}{N\left[1-\delta-\frac{\beta \gamma(a+b)}{N}-\frac{\beta(1-\delta) c \gamma}{N(1-\delta-\beta \gamma)}\right]}$

Ce terme $X$ est forcément positif et inférieur à 1 pour une raison de stabilité de l'équation $(16)^{10}$.

\subsection{Taux d'imposition et durée de formation à l'équilibre}

Le programme de maximisation de l'utilité que résoud l'Etat sous contrainte peut se réécrire ainsi :

$$
\begin{aligned}
\underset{n, \tau, \bar{\tau}}{\operatorname{Max}} U\left(c_{1}, h_{1}\right) & =c_{1}^{\alpha} h_{1}^{1-\alpha} \\
s / c y_{1} & =A_{1} h_{1}^{\beta} n^{1-\beta} \\
c_{1} & =(1-\tau) y_{1} \\
h_{1} & =h_{1}\left(\tau, \bar{\tau}, n, \theta, A_{1}, A_{2}, a, b, c, N, \alpha, \beta, \gamma, \delta\right)
\end{aligned}
$$

\footnotetext{
${ }^{10} \mathrm{Si}$ nous considérons $(a+b)$ proche de 0 , alors $(1-\delta-2 \beta \gamma)$ doit être supérieur à 0 car s'il était nul, nous serions dans un cas de croissance endogène (voir d'Autume, 1994). Et s'il était négatif, l'équilibre serait en coin avec $\tau=0$ pour une très large plage de valeurs de $(a+b)$ ce qui est impossible.
} 
ce qui nous donne les valeurs d'équilibre suivantes :

$$
\begin{gathered}
\tau=\frac{Z}{\alpha+Z} \\
\text { avec } Z=\frac{(a+b) \gamma(\alpha \beta+1-\alpha)}{\frac{N(1-\delta)(1-\delta-2 \beta \gamma)+\beta^{2} \gamma^{2}(a+b)}{1-\delta-\beta \gamma}}=\frac{(a+b) \gamma(\alpha \beta+1-\alpha)}{N(1-\delta)-\beta \gamma\left(a+b+c \frac{(1-\delta)}{1-\delta-\gamma \beta}\right)} \\
=>Z=(a+b)(\alpha \beta+1-\alpha) X
\end{gathered}
$$

Toute augmentation de $(a+b)$ impliquera une hausse de $\tau$. Ce résultat rejoint celui obtenu en l'absence de financement complémentaire. Son interprétation économique repose sur le rendement de la taxation à l'état stationnaire. En effet, l'élasticité de $\tau$ dépend positivement de $(a+b)$ comme le montre l'équation (17). Ici encore les complémentarités jouent leur rôle. Plus le nombre d'agents qui financent l'éducation est grand, plus leurs contributions respectives se complètent pour augmenter le capital humain.

Parallèlement,

$$
\bar{\tau}=\frac{1-\delta-\gamma \beta}{1-\delta}
$$

Le taux d'imposition $\bar{\tau}$ que l'Etat applique aux individus qui déclarent faire partie de la catégorie des plus riches est désormais endogène. Il est constant et indépendant de $a, b$ et $c$ car dans la fonction d'accumulation du capital humain, son élasticité ne dépend pas des catégories de population.

Enfin, la maximisation de l'utilité du plus pauvre par rapport à $n$ conduit à l'expression suivante :

$$
n=\frac{Z^{\prime}}{(\alpha \beta+1-\alpha)+Z^{\prime}}
$$

avec $Z^{\prime}=(1-\beta)\left\{\alpha+\frac{\gamma}{N}(\alpha \beta+1-\alpha) \cdot \frac{N(1-\delta)-\beta \gamma(a+b)}{(1-\delta)[1-\delta-2 \beta \gamma]+\frac{\beta^{2} \gamma^{2}(a+b)}{N}}\right\}$

Pour $(1-\delta-2 \beta \gamma)>0$, toute augmentation de $(a+b)$ impliquera une baisse de $n$ et donc une hausse de $(1-n)$.

En effet, deux mécanismes contradictoires rentrent en jeu dans l'équation (17) du capital humain à l'état stationaire. D'une part, $n$ joue le même rôle que $\tau$ pour les recettes fiscales car une hausse de $\tau$ ou de $n$ accroit les recettes fiscales donc à $a$ constant, une hausse de $b$ accroît $n$ (effet complémentarité). Mais d'autre part, l'Etat a maintenant la possibilité de déterminer 
de manière endogène et optimale la taxation des riches honnêtes, $\bar{\tau}$, sachant qu'il est leader dans l'équilibre de Stackelberg dans lequel les agents riches honnêtes jouent en second pour déterminer leur financement complémentaire. En calculant $\bar{\tau}$, l'Etat va prendre en compte les différentes sources de sousoptimalité (dynamique et effets externes) mais aussi l'effet-revenu négatif : les riches honnêtes investiront moins de manière privée si $\bar{\tau}$ est élevé. Ce faisant, dans l'équation (17), l'élasticité liée au travail des riches honnêtes $(1-\beta)\left[c \frac{(1-\delta)}{1-\delta-\gamma \beta}\right]$ est plus élevée que l'élasticité du travail des pauvres et des riches tricheurs $(1-\beta)(a+b)$.

Or, à $a$ et $N$ constants, si on augmente le nombre de riches tricheurs $b$, on réduit le nombre de riches honnêtes, $c$, qui apparaît dans l'élasticité. Celle-ci se réduit. Augmenter l'évasion fiscale via une hausse de $b$ (et donc une réduction de $c$ ) réduit donc l'effet des complémentarités dans la fonction d'accumulation du capital humain : le temps de travail $n$ diminuera et, de façon complémentaire, la durée d'études $(1-n)$ augmentera.

Dit autrement, pour un $a$ donné, lorsque le nombre de parents ne déclarant pas tous leurs revenus est grand ( $b$ élevé), l'Etat doit imposer une scolarisation obligatoire $(1-n)$ de plus grande ampleur. Ce résultat rejoint celui d'Eckstein et Zilcha (1994) qui, indépendamment de tout phénomène d'évasion fiscale, montrent que l'école obligatoire permet de corriger la sousoptimalité provenant de l'imparfait altruisme des parents vis à vis de leurs enfants. Ici, la scolarisation obligatoire constitue de surcroît une réponse efficace à l'évasion fiscale.

\section{Conclusion}

Les pays en développement, plus encore que les pays développés, sont soumis à une importante évasion fiscale notamment liée à la faiblesse de leurs institutions. Nous supposons que l'Etat, faute de moyens de contrôle suffisants, n'est pas capable d'identifier les fraudeurs au sein de la population. L'objectif de cet article est alors de mobiliser un modèle à générations imbriquées, enrichi de complémentarités, pour montrer dans quelle mesure la quantité et la qualité de l'éducation reçue par la jeune génération sont affectées par ce phénomène. Différentes politiques éducatives sont étudiées selon que les parents financent l'éducation de manière privée ou selon que l'Etat soit en charge de collecter des impôts pour assurer ce financement éducatif. Nous montrons 
que l'évasion fiscale est naturellement source d'inefficacité mais aussi source d'inéquité puisqu'elle conduit à augmenter le taux d'imposition qui s'applique aux plus pauvres. Cependant, l'inefficacité est amoindrie lorsque les parents ont la possibilité de compléter l'éducation publique par une éducation privée individuelle. Dans ce cas, la durée d'études optimale, que l'on peut assimiler à la scolarisation obligatoire déterminée par l'Etat, est croissante avec le nombre de fraudeurs, ce qui vient pallier la baisse de la qualité de l'école publique.

Bien que ces résultats reposent sur une hypothèse forte de reproduction totale des inégalités, les individus se maintenant dans leur catégorie sociale de génération en génération, il nous semble que les mécanismes mis en lumière ici seraient toujours à l'oeuvre dans un modèle plus général.

\section{Bibliographie}

Ahmad E. and Stern N. (1989) : « Taxation for Developing Countries », Handbook of Development Economics, Vol. II, Edited by H. Chenery and T.N. Srinivasan, Elsevier Science Publishers B.V.

d'Autume A. (1994) : « Choix éducatifs, équilibre général et croissance économique », Economie et Prévision, 116, 1994-5, pp 35-48.

Azariadis C. and Drazen A. (1990) : «Threshold Externalities in Economic Development », The Quaterly Journal of Economics, Vol.105, pp.501526.

Basdevant O. (1999) : «Redistribution et croissance optimales dans un modèle d'éducation », Revue économique Vol.5, n 50, pp.985-1007.

Barham V., Boadway R., Marchand M. et Pestieau P. (1995) : « Education and the poverty trap », European Economic Review, Vol.39, pp.12571275 .

Becker G. (1975) : «Human Capital », New York, Columbia University Press for National Bureau of Economic Research.

Becker G. (1981) : « A Treatise on the Family », Cambridge : Harvard University Press.

Becker G.S. and Tomes N. (1976) : « Child Endowments and the Quantity and Quality of Children », Journal of Political Economy, Vol.84, $\mathrm{n}^{\circ} 4$, pp.S143-S162. 
Behrman J.R., Pollak R.A. and Taubman P. (1982) : «Parental Preferences and Provision for Progeny », Journal of Political Economy, Vol.90, $\mathrm{n}^{\circ} 1$, pp.52-73.

Ben-Porath Y. (1967) : « The Production of Human Capital and the Life Cycle of Earnings», Journal of Political Economy, Vol.75, pp.352-365.

Bénabou R. (1996) : « Heterogeneity, Stratification, and Growth : Macroeconomic Implications of Community Structure and School Finance », American Economic Review, Vol.86, n³, pp.584-609.

Böhme M. and Thiele R. (2011) : "Is the Informal Sector Constrained from the Demand Side? Evidence for Six West African Capitals", World Development, Vol. 40, No. 7, pp. 1369-1381.

Bourguignon F. (2000) : « Redistribution et développement", in Conseil d'Analyse Economique, Développement, La documentation française, Paris, pp.11-42.

Diamond P. (1965) : « National Debt in a Neoclassical Growth Model », American Economic Review, Vol.55, n5, pp.1126-1150.

Eckstein Z. and Zilcha I. (1994) : "The effects of compulsory schooling on growth, income distribution and welfare", Journal of Public Economics, 54, pp.339-359.

Epple D. and Romano R.E. (1996a) : « Public Provision of Private Goods », Journal of Political Economy, Vol.104, nº 1, pp.57-84.

Epple D. and Romano R.E. (1996b) : « Ends against the Middle : Determining Public Service Provision when there are Private Alternatives », Journal of Public Economics, Vol.62, pp.297-325.

Epple D. and Romano R.E. (1998) : " Competition Between Private and Public Schools, Vouchers, and Peer-Group Effects », American Economic Review, Vol.88, n 1 , pp.33-62.

Fernandez R. and Rogerson R. (1995) : " On the Political Economy of Education Subsidies », Review of Economic Studies, Vol.62, pp.249-262.

Gérard-Varet, L.A. , Kolm S.C. and J. Mercier Ythier (ed) (2000) "The economics of reciprocity, giving and altruism" Palgrave Mac Millan.

Galor O. and Zeira J. (1993) : «Income Distribution and Macroeconomics », The Review of Economic Studies, Vol.60, n²02, pp.35-52.

Glomm G. (1997) : " Parental choice of human capital investment », Journal of Development Economics, Vol.53, pp.99-114.

Glomm G. and Ravikumar B. (1992) : «Public versus Private Investment in Human Capital : Endogenous Growth and Income Inequality », Journal of Political Economy, Vol.100, n4, pp.819-833. 
Gunther I., Marouani M-A. and Raffinot M. (2007) : « La croissance propauvres au Mali », Agence Française de Développement, Notes et Documents $\mathrm{n}^{\circ} 32$.

Lucas R.E. (1988) : « On the Mechanics of Development Planning », Journal of Monetary Economics, Vol.22, ${ }^{\circ} 1$, pp.3-42.

Pourcet G. (1995) : «Instabilités et structuration du secteur informel » in Ph. Hugon, G. Pourcet et S. Quiers-Valette, «L'Afrique des incertitudes », IEDES, Collection Tiers-Monde, Presses Universitaires de France, Paris, pp.200-220.

Roubaud F. (1994) : «L'économie informelle au Mexique. De la sphère domestique à la dynamique macro-économique », Karthala - ORSTOM, Paris.

Saint-Paul G. and Verdier T. (1993) : « Education, Democracy and Growth », Journal of Development Economics, Vol.42, pp.399-407.

Schultz T.W. (1963) : « The Economic Value of Education », Columbia University Press, New York.

Slemrod J.and Yitzhaki S.(2002) : "Tax Avoidance, Evasion, and Administration", Handbook of Public Economics, Volume 3, Edited by A.J. Auerbach and M. Feldstein, Elsevier science B.V.

Tanzi V. (1983) : "The underground economy", Finance and Development, p.10-13.

World Bank (2014), World Development Indicators. 